\title{
A Quasi-Yagi Antenna Backed by a Jerusalem Cross Frequency Selective Surface
}

\author{
Sergio E. Melais, ${ }^{1}$ David Cure, ${ }^{2}$ and Thomas M. Weller ${ }^{2}$ \\ ${ }^{1}$ Tampa Microwave, Tampa, FL 33626, USA \\ ${ }^{2}$ Department of Electrical Engineering, University of South Florida, Tampa, FL 33620, USA
}

Correspondence should be addressed to Sergio E. Melais; semelais@gmail.com

Received 6 June 2012; Accepted 14 January 2013

Academic Editor: Walter De Raedt

Copyright (C) 2013 Sergio E. Melais et al. This is an open access article distributed under the Creative Commons Attribution License, which permits unrestricted use, distribution, and reproduction in any medium, provided the original work is properly cited.

A quasi-Yagi antenna is developed to operate at $2.4 \mathrm{GHz}$ (ISM band) presenting a low profile and off-axis radiation when packaged over a metal ground plane. The off-axis radiation is realized by incorporating a Jerusalem cross frequency selective surface (JC-FSS) as the ground plane for the antenna. A JC-FSS is preferred because of its frequency stability in the operating band for a large angular spectrum $\left(\approx 70^{\circ}\right)$ of TE- and TM-polarized incident waves. In this research, the substrate of the antenna flush-mounted on top of the FSS is added to the JC-FSS model and allows for a smaller cell grid. The prepared quasi-Yagi antenna over the JC-FSS offered $260 \mathrm{MHz}$ of functional bandwidth and $54^{\circ}$ of beam tilt towards the end-fire direction. To the best of the authors' knowledge this is the first instance that these two structures are combined for off-axis radiation. Additionally, to support the preferred use of the JC-FSS, the quasi-Yagi is backed by a square patch (SP) FSS for comparison purposes.

\section{Introduction}

The work presented in this paper introduces a quasi-Yagi antenna over a metal reflector with off-axis radiation at $2.4 \mathrm{GHz}$ (ISM band). The main application of interest for this antenna is on sensor nodes comprising a wireless sensor network inside a multipath rich environment such as an aircraft fuselage. In practice these antennas would be mounted atop the metal package of a sensor node and used to communicate preferentially toward the front or rear of the aircraft. The packaging of the antenna over a metal ground plane presents a challenge, however, as this configuration results in undesired phase reflections $\left(1 e^{j 180^{\circ}}\right)$ and image currents from the ground canceling the current of the antenna, degrading its operational bandwidth, and tilting the beam away from the end-fire direction. A previous solution was proposed in [1] which consisted of displacing the metal reflector from the antenna by a suitable distance $\left(0.19 \lambda_{g}=7.5 \mathrm{~mm}\right)$. Though off-axis radiation of $40^{\circ}$ was achieved, the arrangement resulted in an inherently high profile.

The configuration proposed herein introduces a new alternative to [1] as it packages the quasi-Yagi antenna over a high impedance surface (HIS) or electromagnetic band-gap (EBG) structure. The HIS eliminates the out-ofphase reflections generated at the ground from radiating to the antenna and supports the radiation of leaky TE waves in the frequency region of high impedance. The preferred HIS configuration used here is the Jerusalem cross frequency selective surface (JC-FSS) from [2], because of its compact size, numerous parameters for tuning, and its stability over the band gap for a large angular $\left(<70^{\circ}\right)$ spectrum of TE and TM incident waves. The unique feature in [2] is the addition of the antenna substrate layer into the JC-FSS model, allowing for a smaller FSS grid.

The use of an HIS to back existing antenna technologies presents advantages such as preserving a considerable fraction of the antenna bandwidth while reducing profile, improving gain, reducing back radiation, and increasing radiation efficiency. Work described in [3] for an Archimedean spiral antenna over a square patch EBG (versus the conventional $0.25 \lambda$ grounded cavity) offered an overall antenna/EBG profile reduction $>69 \%$ and preserved the bandwidth by $71 \%$ without losing directive gain. Another example, developed in [4], shows a wearable dual-band $(2.4$ and $5.1 \mathrm{GHz})$ coplanar patch antenna combined with a square patch EBG made of common clothing fabrics. The EBG used here improved 


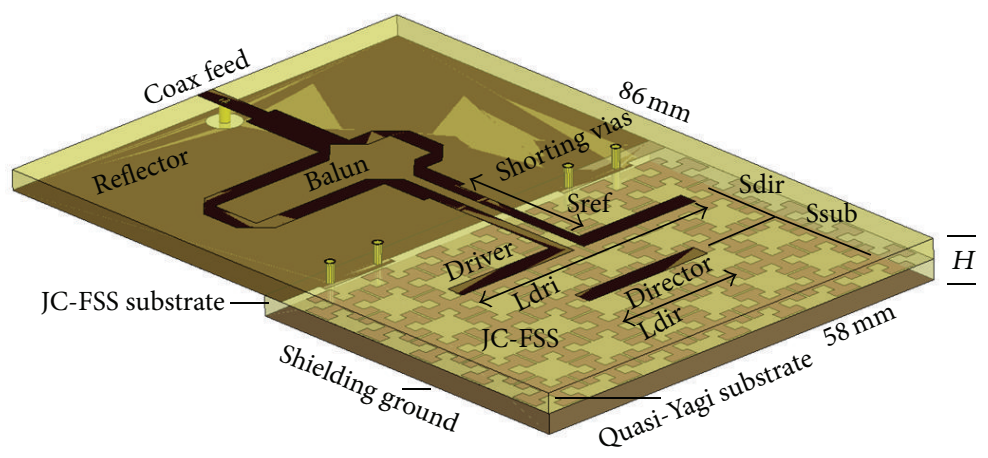

FIGURE 1: Quasi-Yagi antenna over Jerusalem cross frequency selective surface. The beam tilt is measured along the theta direction, aligned with the feed line.

antenna gain $(3 \mathrm{~dB})$ and reduced back radiation into the body $(>10 \mathrm{~dB})$. An equivalent design in [5] provides the same characteristics (high gain and low back radiation), but it used a nonconformal material and the EBG arrangement consisted of a Jerusalem cross-slot array. An example published in [6] shows a flared dipole with quasi end-fire radiation at $3 \mathrm{GHz}$ over an HIS consisting of an array of hexagonal grids (thumbtacks). The amount of angular beam tilting towards the endfire direction depends on the impedance of the HIS, which can be tuned in real time by a variety of methods (e.g., electronically, using varactor diodes [7]). Finally, work presented in [8] illustrates a broadband diamond dipole antenna over a Jerusalem cross frequency selective surface. The bandwidth for the combined structure extends from 5 to $11 \mathrm{GHz}$ with high gain $(>6 \mathrm{~dB})$ from 3.7 to $6.8 \mathrm{GHz}$ and a nondisturbed (without unwanted nulls) directive pattern up to $7 \mathrm{GHz}$.

As suggested above, the majority of existing antenna/EBG configurations present radiation in the broadside direction. The design prepared here combines for the first time a quasiYagi antenna with a JC-FSS over a metal ground with radiation directed toward the end-fire direction. The combined structure has an overall profile of $5 \mathrm{~mm}\left(0.125 \lambda_{g}\right)$, functional bandwidth extending from 2.29 to $2.55 \mathrm{GHz}$, and $54^{\circ}$ of beam tilt towards the end-fire direction. In comparison to [1] the new design provides $33 \%$ reduced profile and $14^{\circ}$ of additional beam tilting. Also, in comparison with a conventional quasiYagi antenna of the same substrate thickness $(5 \mathrm{~mm})$ but lacking the HIS layer, the proposed design offers wider bandwidth $(2.24-2.46 \mathrm{GHz}$ versus $2.35 \mathrm{GHz})$ and $27^{\circ}$ more beam tilting.

The following sections present the antenna design, the derivation of the JC-FSS model with the antenna substrate, and comparisons between simulated and measured results for the combined structure. Also, the performance of the quasiYagi antenna when backed by a square patch FSS is shown to highlight the advantages of the JC-FSS.

\section{Quasi-Yagi Antenna}

2.1. Design Characteristics. A quasi-Yagi antenna, such as that in [9], consists of an array of dipoles printed on a substrate and fed by a microstrip to coplanar strip-line (CPS) transition. The transition is used as a transformer to connect the unbalanced microstrip input line to the balanced (CPS)
TABLE 1: Optimized dimensions of antenna elements.

\begin{tabular}{lcc}
\hline Antenna Element (dimensions in mm.) & {$[1]$} & QY-JCFSS \\
\hline Length of CPS (Sref) & 18.33 & 20.5 \\
Length of driver (Ldri) & 34 & 35.5 \\
Length of director (Ldir) & 18 & 18 \\
Separation of driver and director (Sdir) & 16 & 11.16 \\
Separation of director and end of substrate & 10.83 & 16 \\
(Ssub) & 7.5 & 5 \\
Height of substrate layer $(\mathrm{H})$ & \\
\hline
\end{tabular}

antenna feed line. In addition, the ground plane from the microstrip transition is used as the reflector element for the array, eliminating the need for a reflecting dipole and resulting in a more compact length $\left(<\lambda_{0} / 2\right)$, along with direct compatibility with microstrip circuitry. Further advantages inherent in the design are mechanical support and planar transmission line compatibility due to the presence of a substrate. The use of a high permittivity substrate means that the antenna will be extremely compact in terms of free space wavelength $\left(\lambda_{0}\right)$. In regard to frequency of operation and radiation pattern, quasi-Yagi antennas are broadband $(\approx 50 \%)$ and radiate in the end-fire direction.

The new feature developed in this research, Figure 1, consists of shielding the antenna with a metal ground. To overcome out-of-phase reflection $\left(1 e^{j 180^{\circ}}\right)$ from the metal ground and surface currents from shorting the director and driver dipoles, a JC-FSS is implemented. The ground planes of the FSS and the truncated microstrip are connected to the same potential through shorting vias. The distance separating the antenna and the FSS was determined based on antenna bandwidth requirements and commercially available substrate thickness options. The effect of this distance is accounted for as a superstrate during the derivation of the closed form equations for the JC-FSS model.

The dimensions for the antenna elements in Figure 1 are optimized from [1] for end-fire radiation and to account for the added JC-FSS through simulations in Ansoft HFSS. The substrate material is RT/Duroid $6010 \mathrm{LM}\left(\varepsilon_{r}=10.2\right)$. The overall size of the antenna is $58 \mathrm{~mm} \times 86 \mathrm{~mm}$. The optimized dimensions for the quasi-Yagi antenna are listed in Table 1. 


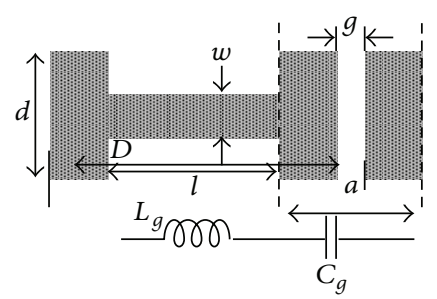

(a)

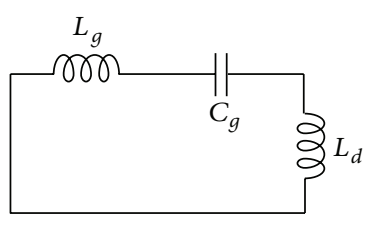

(b)

Figure 2: (a) Equivalent circuit for the self-resonant grid. (b) Series combination of the self resonant grid with the dielectric slab impedance.

The most significant adjustments are the reduction of the driver to director separation (30\%) and the decrease of the overall substrate profile (33\%).

2.2. Jerusalem Cross Frequency Selective Surface. The JC-FSS implemented here was previously developed by the authors in [2]. The design offers in-phase reflection $\left(1 e^{j 0^{\circ}}\right)$ for an operational band extending from 2.39 to $2.5 \mathrm{GHz}$ at normal incidence. In addition, at the center frequency $(2.45 \mathrm{GHz})$ the JC-FSS offers frequency stability for a large angular spectrum $\left(>70^{\circ}\right)$ for both TE- and TM-polarized incident waves. As previously stated, the main feature from the FSS in [2] is the addition of the antenna substrate into the JC-FSS model, which decreases the center frequency of the high impedance band from $3.2 \mathrm{GHz}$ to $2.45 \mathrm{GHz}$ for the same FSS dimensions.

2.2.1. Surface Waves on a Metal Surface versus a Textured Surface. The properties of surface waves on a metal surface versus a textured surface are compared herein, to explain the use of the latter in the quasi-Yagi antenna. If the radiating element is placed near the ground plane, it will generate currents that propagate along the metal sheet. Any break or discontinuity (e.g., the edge of board) on the flat surface will promote radiation from that location. The result is a destructive interference which cancels the radiation from the antenna and decreases the radiation efficiency. By adding a special texture to a metal surface, it is possible to suppress surface currents over a range of frequencies (band gap). As discussed in [10], the electromagnetic properties of the structure can be described by a single value, the surface impedance, if the period of the textured surface is much smaller than the wavelength in the dielectric media $\left(\lambda_{d}\right)$. A smooth or flat conductive sheet has low surface impedance, while a textured surface can be engineered to have high surface impedance.

The fields radiated by the quasi-Yagi antenna over the FSS are a combination of those produced by the antenna elements themselves and those that exist due to the presence of the FSS. For a quasi-Yagi antenna without a ground or underlying structure, the radiated fields are TE with respect to the substrate surface and the direction of propagation (end fire). If a conventional ground was placed beneath the antenna, TM surface wave propagation is possible; however, these waves are unlikely to be excited by the antenna elements due to their orientation. The main problem with this configuration is field cancelation due to image currents. With the textured
FSS layer beneath the antenna both TE and TM wave propagations are possible. However, TE wave excitation is dominant because of the orientation of the antenna elements. When the surface impedance is large, these TE waves are leaky and radiate readily, causing the overall radiation pattern to tilt away from broadside. The low cross-polarization levels achieved with the antenna presented herein support the conclusion that TM surface wave radiation is not significant.

2.2.2. Derivation of the JC-FSS Model. The JC-FSS of this work is effectively modeled by a parallel resonant LC circuit following the condition that the grid period is smaller than the wavelength $\left(D \ll \lambda_{g}\right)$. The LC model consists of the parallel combination of the self-resonant grid impedance $\left(Z_{q}\right)$, which represents a strip, with the grounded dielectric slab impedance $\left(Z_{d}\right)$. Figure $2(a)$ shows that $Z_{g}$ can be expanded into the series combination of the narrow strip impedance and the edge impedance between end loading strips. The narrow strip impedance is mostly inductive $\left(L_{g}\right)$ and is derived from Telegrapher's equations or from the stepped impedance equations from [11]. The equation for the grid inductance is written as

$$
L_{g}=\frac{Z_{o} \beta \ell}{\omega}
$$

The impedance between end loading plates is mostly capacitive $\left(C_{g}\right)$ and is a result of the charge builtup between plates [10]. This capacitance is given by

$$
C_{g}=\frac{2 d}{\pi} \varepsilon_{o} \varepsilon_{\mathrm{eff}} \cosh ^{-1}\left(\frac{a}{g}\right),
$$

where $\varepsilon_{\text {eff }}$ is the effective permittivity including the superstrate layer, $d$ is the length of an end loading plate, $g$ is the gap between crosses, and $a$ is the period between adjacent capacitive plates.

As illustrated in Figure 2(b) $Z_{d}$, is mostly inductive $\left(L_{d}\right)$ and is derived from the TEM transmission line equation for a dielectric slab backed by a perfect electric conductor [12]. In (3) $k$ is the wavenumber $\omega \sqrt{\mu_{0} \varepsilon_{0}} \sqrt{\varepsilon_{r}}, h$ is the dielectric height, and $\eta_{0}$ is the intrinsic impedance in free space:

$$
L_{d}=\frac{\eta_{o}}{\varepsilon_{r}} \frac{\tan (k h)}{\omega} .
$$


From the parallel LC circuit in Figure 2(b) the equivalent surface impedance is calculated by

$$
Z_{s}=j \omega L_{d} \frac{1-\omega^{2} L_{g} C_{g}}{1-\omega^{2}\left(L_{g}+L_{d}\right) \cdot C_{g}} .
$$

The resonant frequency can then be derived by equating the denominator from (4) to zero which results in

$$
f_{r}=\frac{1}{2 \pi \sqrt{\left(L_{g}+L_{d}\right) \cdot C_{g}}} .
$$

The bandwidth is obtained by dividing the equivalent impedance of the JC-FSS by $\eta_{o}$ and following the criteria in $[12,13]$ that the phase of the reflection coefficient should fall between $\pm 0.25 \pi$.

The dimensions for the JC-FSS are listed in [2]. Both dielectric layers have a high relative permittivity $\left(\varepsilon_{r}=10.2\right)$ offering better angular stability and smaller dimensions at low resonant frequencies. Each dielectric layer is relatively thick $(2.5 \mathrm{~mm})$, increasing the inductance $\left(L_{d}\right)$ of the equivalent surface impedance (4). The use of a small gap $(g=0.32 \mathrm{~mm})$ between crosses leads to larger edge capacitance $\left(C_{g}\right)$ in the surface impedance.

2.2.3. Impact of Superstrate on JC-FSS Model. The most attractive trait from the JC-FSS in [2], compared to others [13], is that the antenna substrate (or superstrate) is included into the JC-FSS model. Here, the impinging wave is excited on top of this layer, (Figure 3). The equivalent reflection coefficient $\left(\Gamma_{\text {IN }}\right)$ for the structure at oblique incidence for TEand TM-polarized waves is the result of the combination of the reflected waves from the two dielectric layers and ground. Also, the addition of the superstrate varies the effective permittivity $\left(\varepsilon_{\text {eff }}\right)$ between layers from 7.2 to 9.7 , which is considered during the derivation of the grid capacitance in (2).

The reflection coefficient for TE and TM waves at the JCFSS/superstrate boundary is calculated by combining (4) and $\eta_{1}=\eta_{o} / \sqrt{10.2}$ with

$$
\begin{gathered}
\Gamma_{s}^{\mathrm{TE}}=\frac{Z_{s} \cdot \cos \left(\theta^{1}\right)-\eta_{1} \cdot \cos (\theta)}{Z_{s} \cdot \cos \left(\theta^{1}\right)+\eta_{1} \cdot \cos (\theta)}, \\
\Gamma_{s}^{\mathrm{TM}}=\frac{Z_{s} \cdot \cos (\theta)-\eta_{1} \cdot \cos \left(\theta^{1}\right)}{Z_{s} \cdot \cos (\theta)+\eta_{1} \cdot \cos \left(\theta^{1}\right)},
\end{gathered}
$$

where the incident $\left(\theta^{1}\right)$ and refracted $(\theta)$ angles have minimum effect on the phase of the TE and TM reflected wave $\left(\angle \Gamma_{s}^{\circ}\right)$ for the frequency range in which the JC-FSS exhibits high impedance, resulting in angular stability.

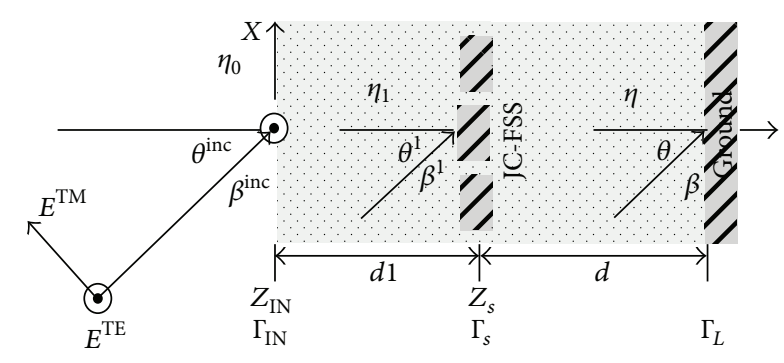

FIGURE 3: TE- and TM-polarized waves incident on the superstrate layer covering the FSS.

Next, the reflection coefficient at the free-space/superstrate boundary for TE and TM waves is derived. First the input impedance is found for the wave reflected from the FSS:

$$
\begin{aligned}
& \mathrm{Z}_{\mathrm{IN}}{ }^{\mathrm{TE}}=\eta_{1} \cdot \frac{1+\Gamma_{s}{ }^{\mathrm{TE}} e^{-j \cdot 2 \cdot \beta^{1} d 1}}{1-\Gamma_{s}{ }^{\mathrm{TE}} e^{-j \cdot 2 \cdot \beta^{1} d 1}}, \\
& \mathrm{Z}_{\mathrm{IN}}{ }^{\mathrm{TM}}=\eta_{1} \cdot \frac{1+\Gamma_{s}^{\mathrm{TM}} e^{-j \cdot 2 \cdot \beta^{1} d 1}}{1-\Gamma_{s}^{\mathrm{TM}} e^{-j \cdot 2 \cdot \beta^{1} d 1}},
\end{aligned}
$$

and the results are included in

$$
\begin{gathered}
\Gamma_{\mathrm{IN}}{ }^{\mathrm{TE}}=\frac{\mathrm{Z}_{\mathrm{IN}}{ }^{\mathrm{TE}} \cdot \cos \left(\theta^{\text {inc }}\right)-\eta_{0} \cdot \cos \left(\theta^{1}\right)}{Z_{\mathrm{IN}}{ }^{\mathrm{TE}} \cdot \cos \left(\theta^{\text {inc }}\right)+\eta_{0} \cdot \cos \left(\theta^{1}\right)}, \\
\Gamma_{\mathrm{IN}}{ }^{\mathrm{TM}}=\frac{Z_{\mathrm{IN}}{ }^{\mathrm{TM}} \cdot \cos \left(\theta^{1}\right)-\eta_{0} \cdot \cos \left(\theta^{\text {inc }}\right)}{Z_{\mathrm{IN}}{ }^{\mathrm{TM}} \cdot \cos \left(\theta^{1}\right)+\eta_{0} \cdot \cos \left(\theta^{\text {inc }}\right)} .
\end{gathered}
$$

The relation between the angle of incidence and refraction at the boundary of each layer is determined from Snell's law of refraction in [14]. This equation demonstrates that the addition of the superstrate layer reduces the angle of incidence for the wave impinging on the JC-FSS. For example, a travelling wave with an angle of incidence of $60^{\circ}$ at the superstrate surface has an angle of refraction of $15^{\circ}$. This angle of refraction will be the angle of incidence for the FSS.

A comparison is performed on the design of a JCFSS including the superstrate versus a design without the superstrate. For simplicity the evaluation is carried out for a wave with normal incidence. At normal incidence $\Gamma_{I N}$ is independent of the polarization of the incident wave since the $E$ and $H$ fields are both tangential to the boundary. Simulation results in Figure 4 demonstrate that the superstrate shifts the center frequency down to the desired band from 3.2 to $2.45 \mathrm{GHz}(750 \mathrm{MHz})$. This analysis supports the importance of accounting for any additional layer covering the JC-FSS during the FSS closed-form modeling to prevent undesired frequency shifts. In addition, an overall cell size reduction has been achieved by considering the extra layer; an equivalent cell size for $3 \mathrm{GHz}$, with no superstrate, is used at $2.4 \mathrm{GHz}$ with superstrate. 


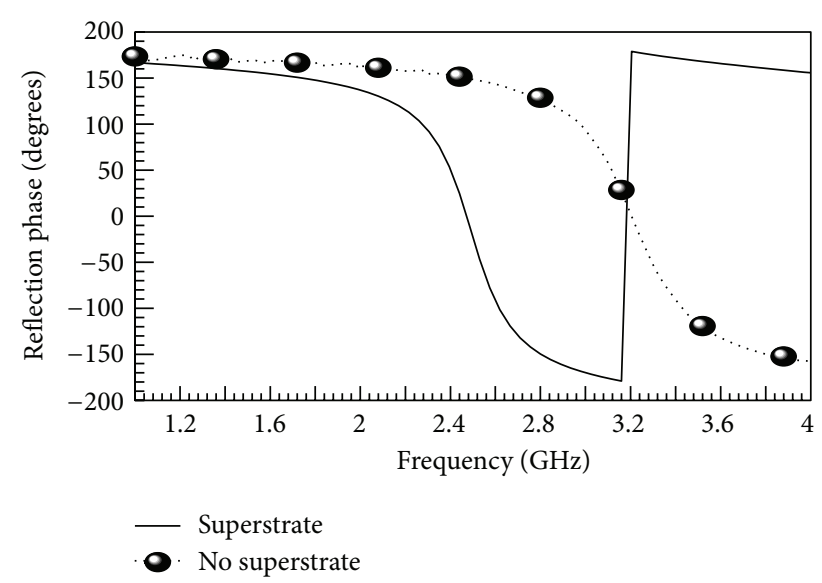

FIGURE 4: Reflection phase (deg.) versus frequency at normal incidence for a JC-FSS with and without a superstrate.

\section{Simulation and Measurement Results}

A comparison between measured and simulated data on return loss and radiation pattern is presented in this section for the quasi-Yagi antenna backed by the JC-FSS. Additionally, these same results are compared against data for a conventional grounded quasi-Yagi antenna of the same substrate height $(5 \mathrm{~mm})$ and the quasi-Yagi design presented in [1]. Finally, a comparison is made between the JC-FSS design and one using a square patch FSS (SP-FSS).

The simulated and measured reflection coefficients for the quasi-Yagi antenna over the JC-FSS are compared in Figure 5. The simulated data demonstrate an operational bandwidth from 2.24 to $2.46 \mathrm{GHz}$. However, the measured frequency band is shifted up in frequency, and the response exhibits undesired reflections in the 2.4 to $2.5 \mathrm{GHz}$ frequency range. These effects are a result of the sensitivity of the JCFSS to small air gaps from the adhesive used to attach the antenna substrate to the JC-FSS substrate. This explanation is confirmed through simulations, (Figure 6), where the bond line is approximated by a 1.5 mil air gap between layers. Furthermore, a small air gap will also affect $\varepsilon_{\text {eff }}$, disturbing the impedances of the grid design.

The results for the simulated and measured normalized $\mathrm{H}$-plane patterns at $2.45 \mathrm{GHz}$ are illustrated in Figures 7 and 8 , respectively. The simulated and measured results demonstrate a copolarized $\mathrm{H}$-plane (H-CPOL) pattern with beam tilt of $45^{\circ}$ and $54^{\circ}$, respectively, towards the end-fire direction. At $45^{\circ}$ the simulated beam peak is 1 and $3 \mathrm{~dB}$ larger than at the end-fire $\left(\theta=90^{\circ}\right)$ and broadside $\left(\theta=0^{\circ}\right)$ directions. Correspondingly the measured beam peak at $54^{\circ}$ is 2 and $3 \mathrm{~dB}$ larger than at $\theta=90^{\circ}$ and $0^{\circ}$.

The simulated and measured H-plane cross-polarization ( $\mathrm{H}$-XPOL) levels are -22.6 and $-13 \mathrm{~dB}$. The drastic increase in the measured H-XPOL level is attributed to measurement set-up tolerances, sensitivity of the JC-FSS to large angles of incidence, and to the air gap resulting from the adhesive. If the resonant frequency of the high impedance band moves up enough such that the frequency of interest $(2.45 \mathrm{GHz})$ falls below the band gap, then the surface impedance is

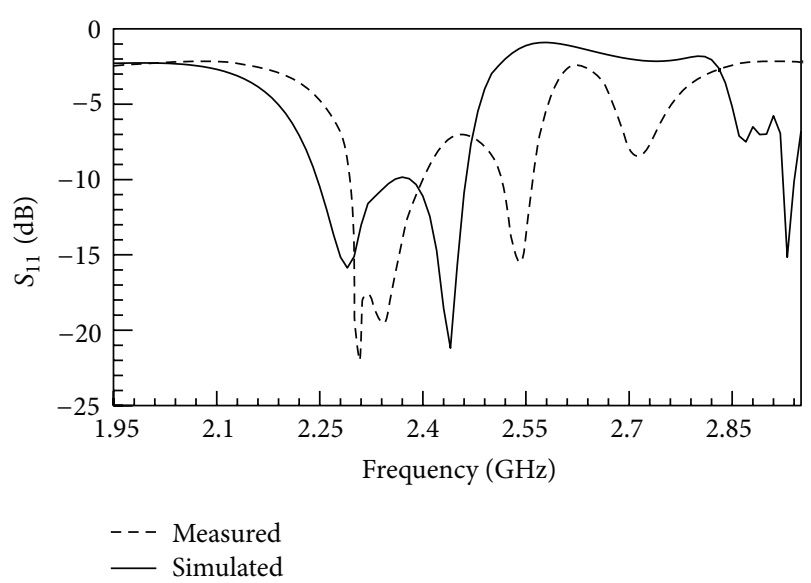

FIGURE 5: Simulated versus measured reflection coefficient for the quasi-Yagi antenna over the JC-FSS. The simulated return loss bandwidth extends from 2.24 to $2.46 \mathrm{GHz}$ while the measured bandwidth extends from 2.29 to $2.55 \mathrm{GHz}$.

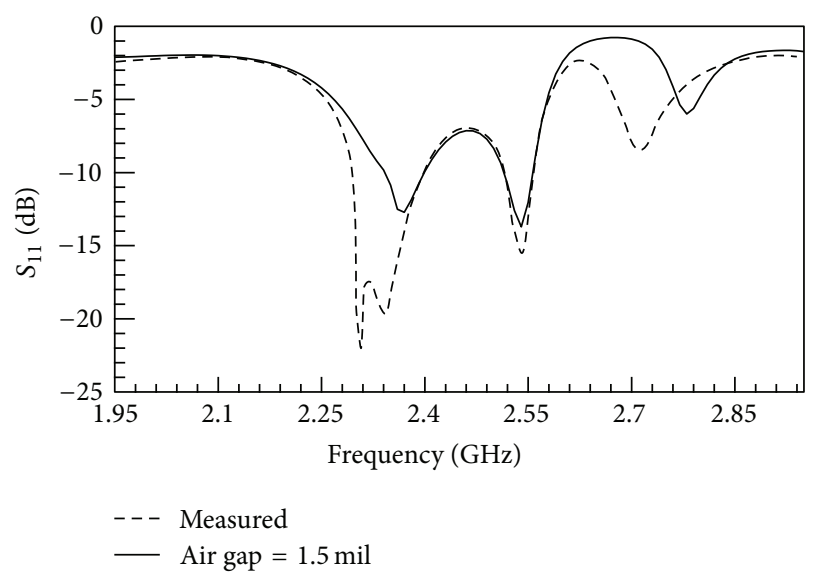

FIGURE 6: Simulation results including a 1.5 mil air gap between substrate layers versus measurement data for the quasi-Yagi antenna over the JC-FSS, to attest the frequency shift on the measured design.

inductive and TM surface waves from the ground radiate readily thereby increasing the X-POL levels [10].

Simulations were performed on a quasi-Yagi antenna printed on a $5 \mathrm{~mm}$ thick grounded dielectric slab in order to assess the impact of including the JC-FSS layer. The comparison presented in Figure 9 shows a drastic improvement of $220 \mathrm{MHz}$ in the return loss bandwidth for the design including the JC-FSS versus the design over the $5 \mathrm{~mm}$ thick grounded slab. A similar evaluation is shown in Figure 10 comparing the copolarization (C-POL) level for the H-plane pattern versus frequency $(2.4,2.45$, and $2.5 \mathrm{GHz})$. For the quasi-Yagi design backed by the JC-FSS, the H-plane CPOL pattern provides an additional $27^{\circ}$ of beam tilt towards the end-fire direction. Furthermore, in the end-fire direction $\left(\theta=90^{\circ}\right)$ the gain increases by $4.5 \mathrm{~dB}$ at $2.4 \mathrm{GHz}, 5.03 \mathrm{~dB}$ at $2.45 \mathrm{GHz}$, and $5.68 \mathrm{~dB}$ at $2.5 \mathrm{GHz}$ relative to the design on the grounded slab. 


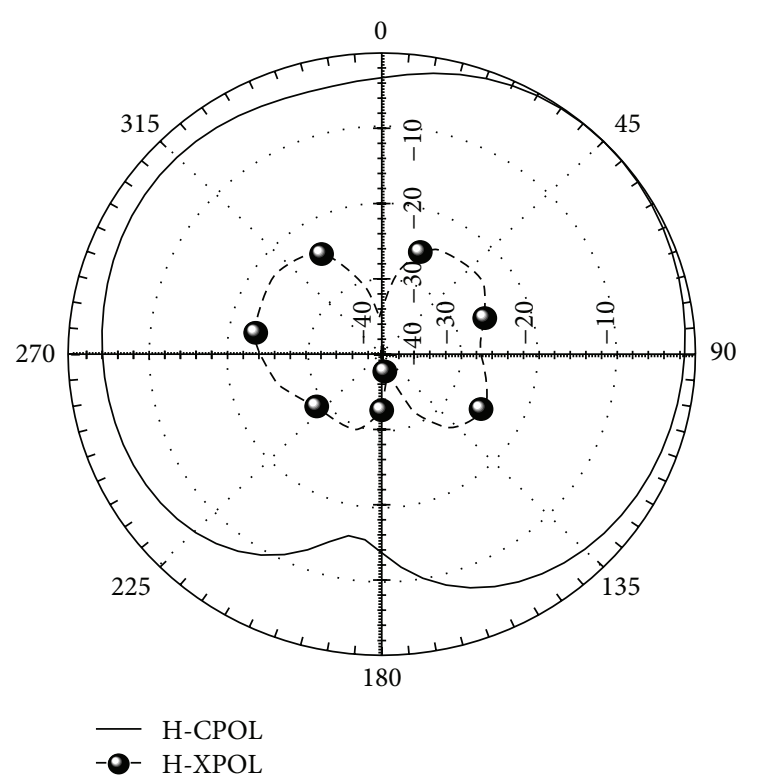

FIgURE 7: Simulation data of the normalized H-plane pattern at $2.45 \mathrm{GHz}$ for the quasi-Yagi antenna over the JC-FSS. The beam peak angle shows a maximum tilt of $45^{\circ}$. The pattern is normalized to the $\mathrm{H}-\mathrm{CPOL}$ value.

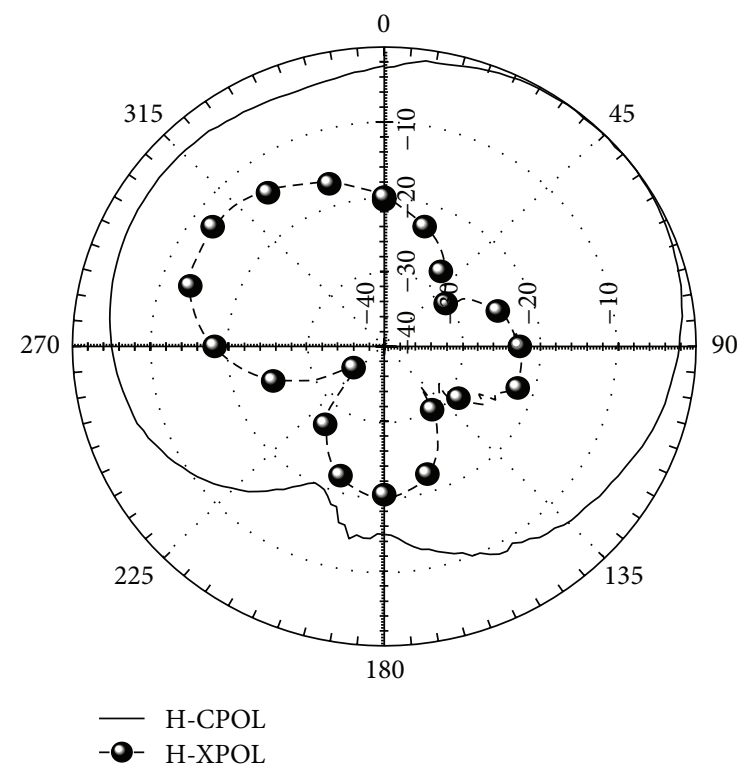

Figure 8: Measurement data for normalized H-plane pattern at $2.45 \mathrm{GHz}$. The maximum beam peak is recorded at $54^{\circ}$. The pattern is normalized to the $\mathrm{H}-\mathrm{CPOL}$ value.

Previous work in [1] presented a quasi-Yagi antenna packaged over a grounded dielectric slab $(7.5 \mathrm{~mm}$ thick) with an operational bandwidth from 2.36 to $2.55 \mathrm{GHz}$ and off-axis radiation of $40^{\circ}$. In comparison to [1], the antenna backed by the JC-FSS presents a wider bandwidth extending from 2.29 to $2.55 \mathrm{GHz}, 14^{\circ}$ of additional beam tilt towards the end-fire direction and an overall profile reduction of $33 \%$.

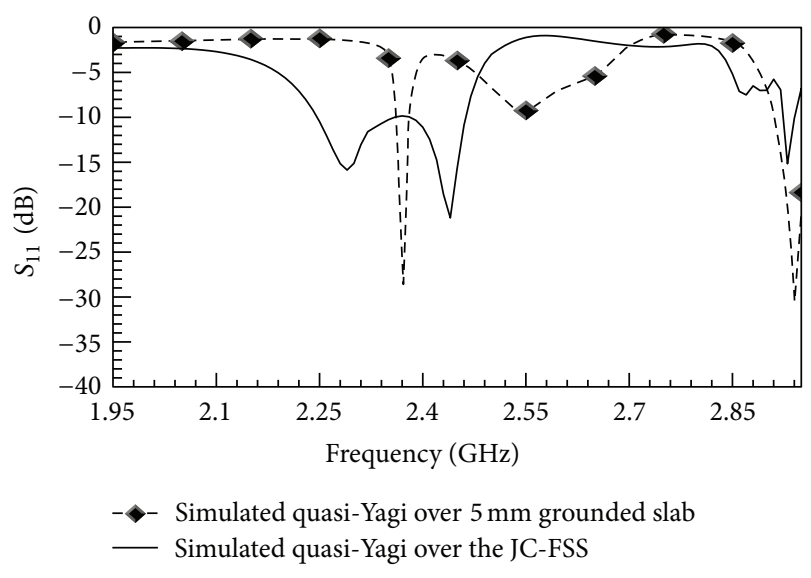

FIGURE 9: Simulation comparing the return loss bandwidth for a quasi-Yagi antenna resting on top of a grounded dielectric slab versus the design on top of the JC-FSS. The simulated bandwidth for the grounded slab is very narrow $(2.35 \mathrm{GHz})$ while the bandwidth of the design that includes the JC-FSS extends from 2.24 to $2.46 \mathrm{GHz}$.

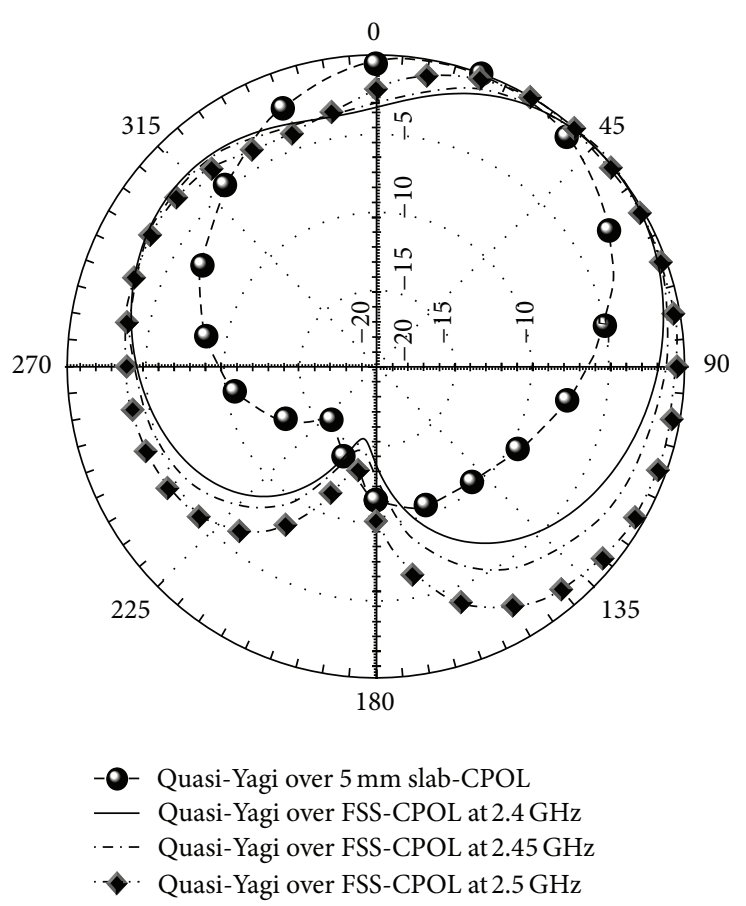

Figure 10: Copolarization of the H-plane pattern at 2.4, 2.45, and $2.5 \mathrm{GHz}$ for the quasi-Yagi design on top of a grounded dielectric slab versus the quasi-Yagi design on top of the JC-FSS.

3.1. Quasi-Yagi Backed by an SP-FSS. In this section the SPFSS derived in [15] is realized as the HIS structure for the quasi-Yagi antenna. The objective is to assess the dependence of the quasi-Yagi on the chosen HIS to promote principal beam tilting towards the off-axis direction. The proposed SP-FSS consists of a periodic cell with length and width of $3 \mathrm{~mm}$ and a gap of $0.1 \mathrm{~mm}$ between adjacent cells. In addition, as suggested by [16], the size of the ground plane beneath 


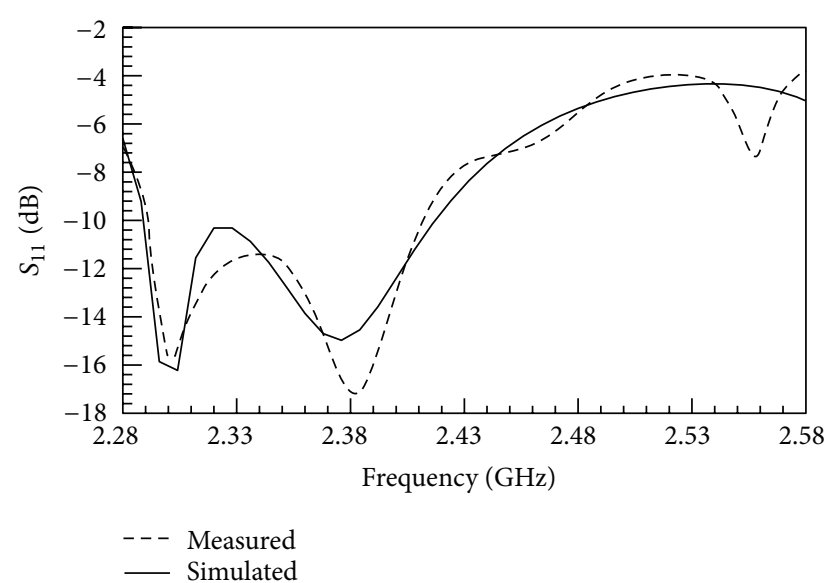

FIGURE 11: Simulated versus measured return loss for the quasi-Yagi antenna over the SP-FSS. The simulated and measured return loss bandwidth extends from 2.28 to $2.43 \mathrm{GHz}$

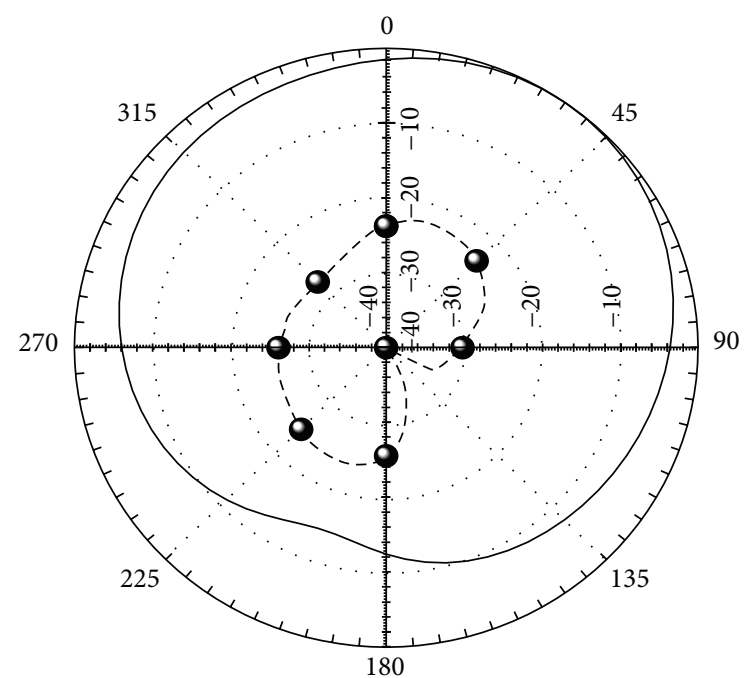

H-CPOL

- $\mathrm{H}-\mathrm{XPOL}$

FIGURE 12: Simulation data for the normalized H-plane pattern at $2.38 \mathrm{GHz}$ for the quasi-Yagi antenna over the SP-FSS. The beam peak angle shows a maximum tilt of $38^{\circ}$. The pattern is normalized to the $\mathrm{H}$-CPOL value.

the director/driver dipoles was kept unchanged from JC-FSS design to avoid the introduction of unwanted resonances in the return loss response.

The SP-FSS design was fabricated and assembled using the same process used for the JC-FSS design, and the measured performance was compared to HFSS simulation data. Figure 11 demonstrates close agreement between the simulated and measured return loss with an operational bandwidth from 2.28 to $2.43 \mathrm{GHz}$. Figures 12 and 13 show the simulated and measured normalized $\mathrm{H}$-plane patterns at $2.38 \mathrm{GHz}$. The simulated and measured results demonstrate a copolarized H-plane (H-CPOL) pattern with beam tilt of $38^{\circ}$ and $35^{\circ}$, respectively, towards the off-axis direction. At $38^{\circ}$ the

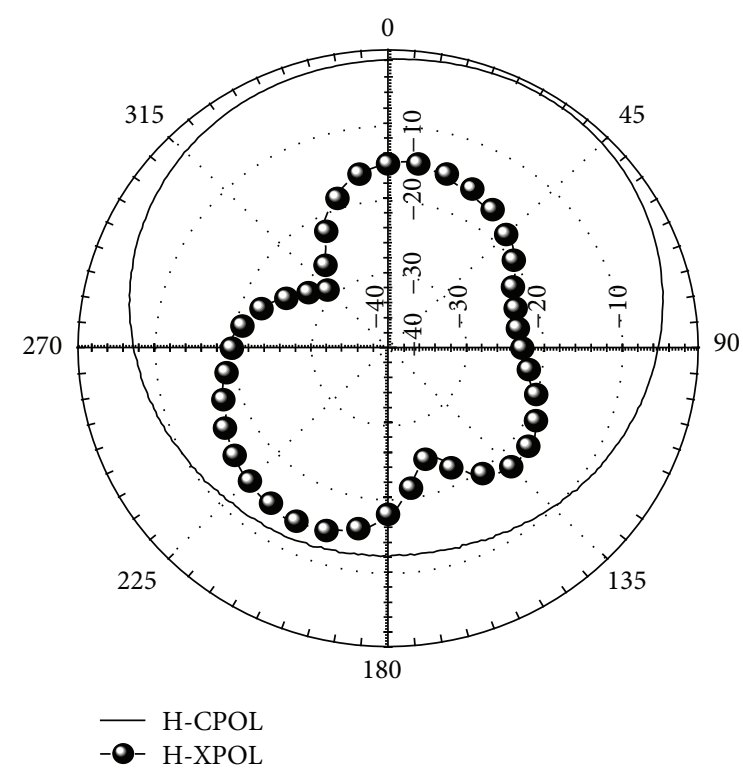

FIGURE 13: Measurement data for normalized H-plane pattern at $2.38 \mathrm{GHz}$. The maximum beam peak is recorded at $35^{\circ}$. The pattern is normalized to the $\mathrm{H}-\mathrm{CPOL}$ value.

simulated beam peak is 1.5 and $3.5 \mathrm{~dB}$ larger than at the endfire $\left(\theta=90^{\circ}\right)$ and broadside $\left(\theta=0^{\circ}\right)$ directions. Correspondingly the measured beam peak at $35^{\circ}$ is 1.3 and $4.2 \mathrm{~dB}$ larger than at $\theta=90^{\circ}$ and $0^{\circ}$. The simulated and measured H-plane cross-polarization (H-XPOL) levels are -25 and $-14 \mathrm{~dB}$.

The presented evaluation of the JC-FSS versus the SP-FSS has demonstrated that the JC-FSS provides additional beam tilting of $19^{\circ}$ towards the off-axis direction. This is the result of the inherent angular stability and high inductance from the JC grid which makes it a preferable shielding candidate for antennas with off-axis radiation.

\section{Conclusion}

A new design for a quasi-Yagi antenna backed by a metal ground and with end-fire-like radiation has been proposed. The design consisted of packaging the antenna over a JCFSS. This is the first time that these two structures are combined for end-fire operation. The results on return loss show an operational bandwidth from 2.29 to $2.55 \mathrm{GHz}$. The $\mathrm{H}$ plane pattern showed beam tilt of $54^{\circ}$ towards the end-fire direction.

In comparison to a design of same substrate height $(5 \mathrm{~mm})$ but without the JC-FSS, the proposed design offers $220 \mathrm{MHz}$ more bandwidth and $27^{\circ}$ of extra beam tilting in the end-fire direction. Furthermore, when compared to the option previously proposed in [1] with the quasi-Yagi antenna placed over a thick grounded slab, the proposed design offers a profile reduction of $33 \%$ and $14^{\circ}$ of additional beam tilt in the end-fire direction. Additionally, the presence of the superstrate above the JC-FSS reduces the physical size of the unit cells by $23 \%$. Finally, the comparison carried out on the quasi-Yagi backed by a SP-FSS has demonstrated that selecting an FSS with inherent angular stability for oblique 
angles of incidence is preferred for antennas with radiation patterns towards the end-fire direction.

\section{Acknowledgments}

The authors wish to express appreciation to Ansoft Corporation and Rogers Corporation for providing software and materials necessary for this work. This work was supported in part by Goodrich Lighting Systems in Oldsmar, Florida.

\section{References}

[1] S. E. Melais and T. M. Weller, "A Quasi Yagi antenna backed by a metal reflector," IEEE Transactions on Antennas and Propagation, vol. 56, no. 12, pp. 3868-3872, 2008.

[2] S. E. Melais and T. M. Weller, "A multilayer Jerusalem cross frequency selective surface," in Proceedings of the IEEE 10th Annual Wireless and Microwave Technology Conference (WAMICON '09), April 2009.

[3] J. M. Bell and M. F. Iskander, "A low-profile archimedean spiral antenna using an EBG ground plane," IEEE Antennas and Wireless Propagation Letters, vol. 3, no. 1, pp. 223-226, 2004.

[4] S. Zhu and R. Langley, "Dual-band wearable antennas over EBG substrate," Electronics Letters, vol. 43, no. 3, pp. 141-143, 2007.

[5] Q. Luo, H. M. Salgado, A. M. Moura, and J. R. Pereira, "Dualband antenna design using an EBG artificial magnetic conductor ground plane," in Proceedings of the Loughborough Antennas and Propagation Conference (LAPC '08), pp. 217-220, Loughborough, UK, March 2008.

[6] J. J. Lee, R. J. Broas, and S. Livingston, "Flush-mounted antennas on Hi-Z ground planes," in Proceedings of the IEEE Antennas and Propagation Society International Symposium, vol. 3, pp. 764-767, June 2002.

[7] D. F. Sievenpiper, J. H. Schaffner, H. Jae Song, R. Y. Loo, and G. Tangonan, "Two-Dimensional Beam Steering Using an Electrically Tunable Impedance Surface," IEEE Transactions on Antennas and Propagation, vol. 51, no. 10I, pp. 2713-2722, 2003.

[8] L. Akhoondzadeh-Asl, P. S. Hall, J. Nourinia, and C. Ghobadi, "Influence of angular stability of EBG structures on low profile dipole antenna performance," in Proceedings of the IEEE International Workshop on Antenna Technology (IWAT '06), pp. 253256, March 2006.

[9] N. Kaneda, Y. Qian, and T. Itoh, "A novel Yagi-Uda dipole array fed by a microstrip-to-CPS transition," in Proceedings of the Asia-Pacific Microwave Conference, pp. 1413-1416, Yokohama, Japan, December 1998.

[10] D. Sievenpiper, High-impedance electromagnetic surfaces [Ph.D. dissertation], Electrical Engineering Department University of California, Los Angeles, Los Angeles, Calif, USA, 1999.

[11] D. M. Pozar, Microwave Engineering, Wiley, New York, NY, USA, 3rd edition, 2005.

[12] M. Hosseini and M. Hakkak, "Characteristics estimation for jerusalem cross-based artificial magnetic conductors," IEEE Antennas and Wireless Propagation Letters, vol. 7, pp. 58-61, 2008.

[13] M. Hosseini, A. Pirhadi, and M. Hakkak, "Design of an AMC with little sensitivity to angle of incidence using an optimized Jerusalem cross FSS," in Proceedings of the IEEE International Workshop on Antenna Technology (IWAT'06), pp. 245-248, New York, NY, USA, March 2006.

[14] C. A. Balanis, Advanced Engineering Electromagnetics, John Wiley \& Sons, 1989.
[15] D. Cure, T. Weller, and F. A. Miranda, "A comparison between Jerusalem Cross and Square Patch Frequency Selective Surfaces for low profile antenna applications," in Proceedings of the IEEE International Conference on Electromagnetics in Advanced Applications (ICEAA '11), pp. 1019-11022, Torino, Italy, September 2011.

[16] F. Costa, O. Luukkonen, C. R. Simovski, A. Monorchio, P. de Maagt, and S. A. Tetryakov, "TE surface wave resonances on high-impedance surface based antennas: analysis and modeling," IEEE Transactions on Antennas and Propagation, vol. 59, no. 10, pp. 3588-3596, 2011. 

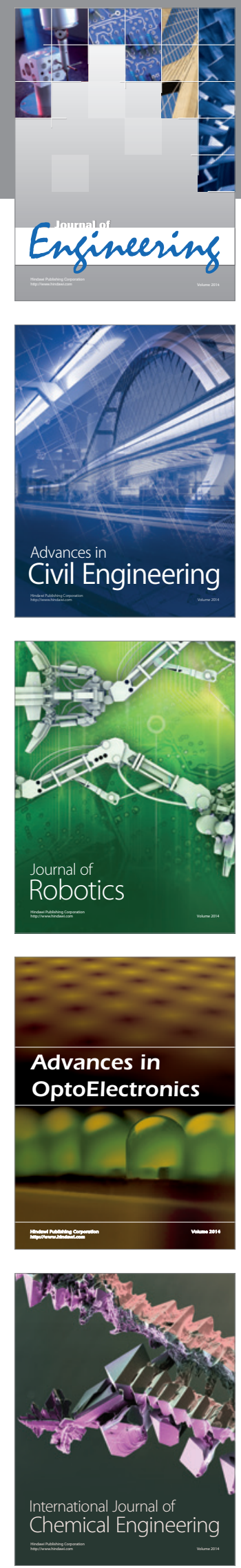

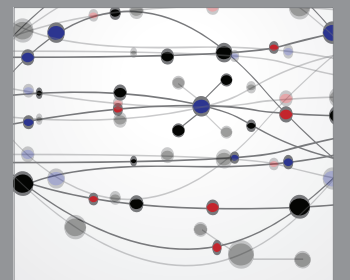

The Scientific World Journal
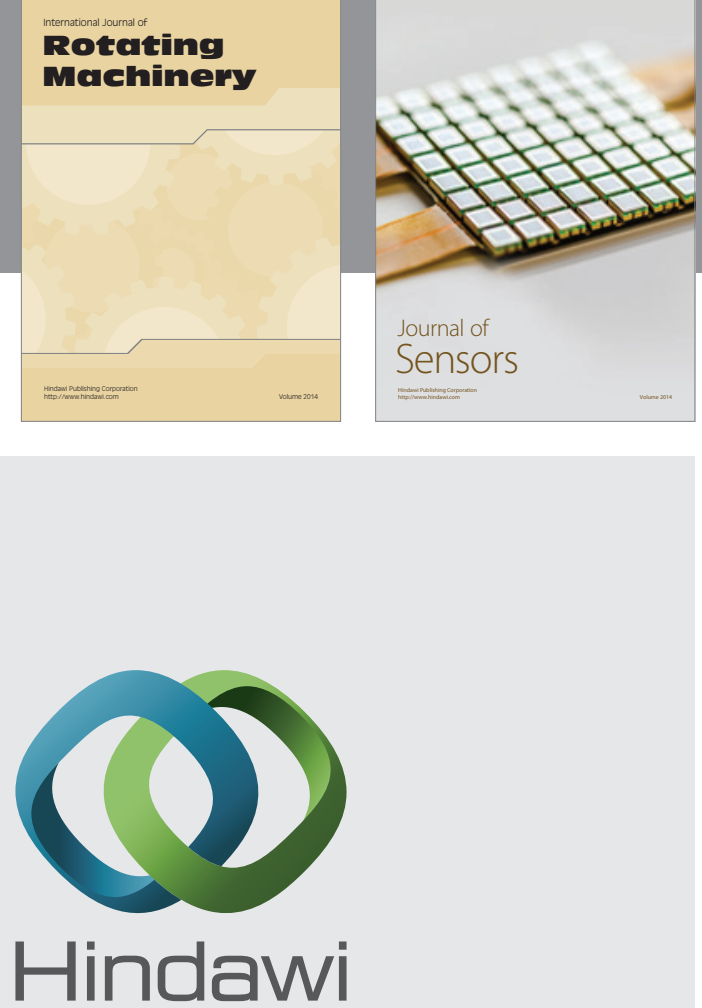

Submit your manuscripts at http://www.hindawi.com
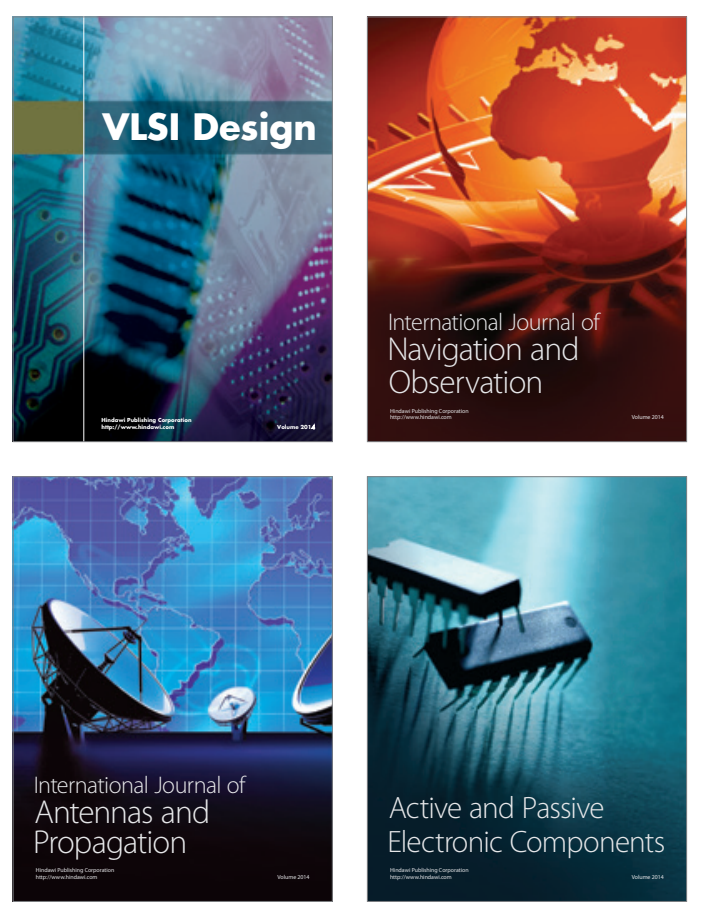
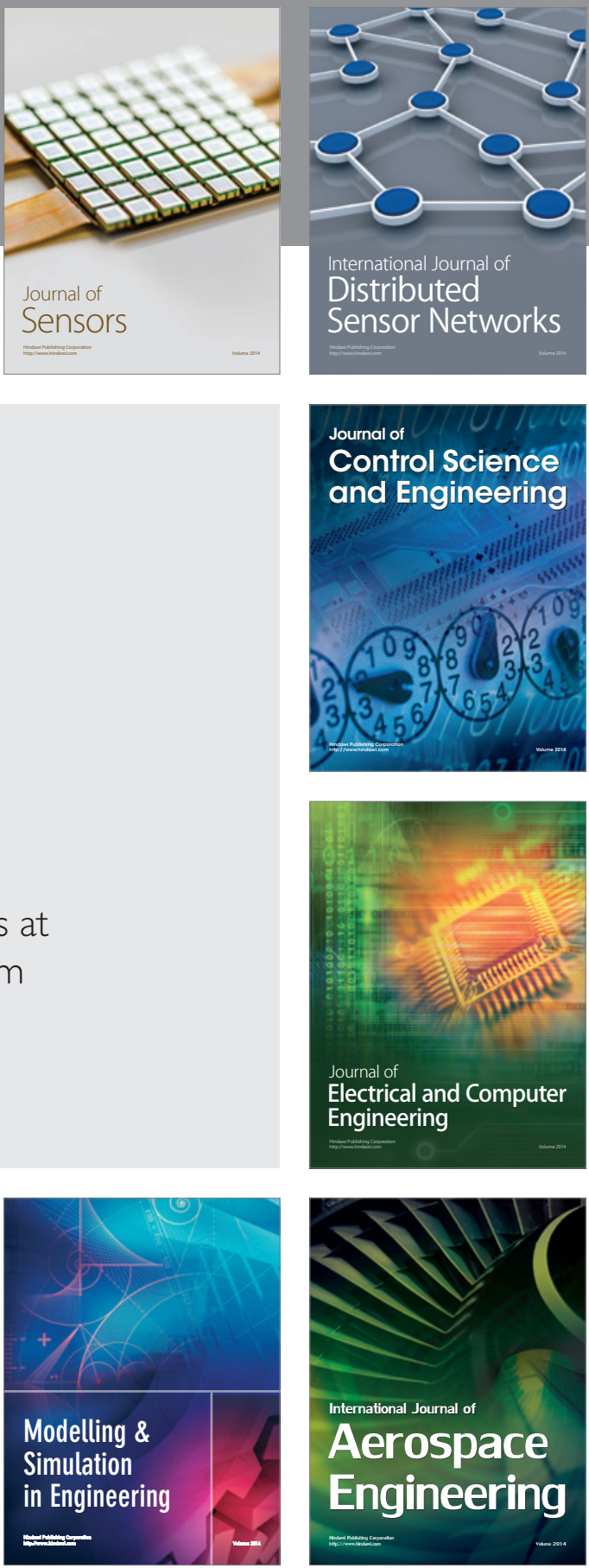

Journal of

Control Science

and Engineering
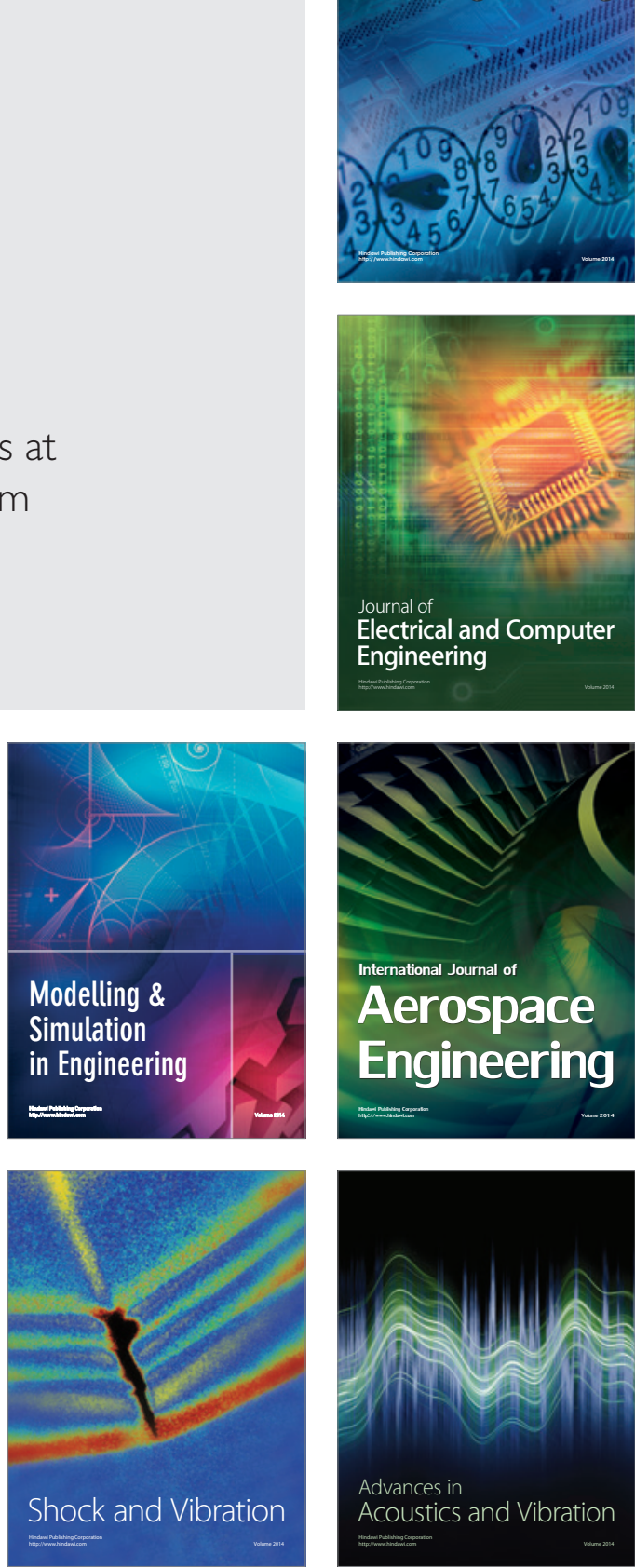